\title{
Post-Routing Redundant Via Insertion and Line End Extension with Via Density Consideration *
}

\author{
Kuang-Yao Lee \\ Department of Computer Science \\ National Tsing Hua University \\ Hsinchu, Taiwan \\ d924347@oz.nthu.edu.tw
}

\author{
Ting-Chi Wang \\ Department of Computer Science \\ National Tsing Hua University \\ Hsinchu, Taiwan \\ tcwang@cs.nthu.edu.tw
}

\author{
Kai-Yuan Chao \\ Intel Corporation \\ Hillsboro, OR 97124 \\ kaiyuan.chao@intel.com
}

\begin{abstract}
Redundant via insertion and line end extension employed in the post-routing stage are two well known and highly recommended techniques to reduce yield loss due to via failure. However, if the amount of inserted redundant vias is not well controlled, it could violate via density rules and adversely worsen the yield and reliability of the design. In this paper, we first study the problem of redundant via insertion, and present two methods to accelerate a state-ofthe-art approach (which is based on a maximum independent set (MIS) formulation) to solve it. We then consider the problem of simultaneous redundant via insertion and line end extension. We formulate the problem as a maximum weighted independent set (MWIS) problem and modify the accelerated MIS-based approach to solve it. Lastly, we investigate the problem of simultaneous redundant via insertion and line end extension subject to the maximum via density rule, and present a two-stage approach for it. In the first stage, we ignore the maximum via density rule, and enhance the MWIS-based approach to find the set of regions which violate the maximum via density rule after performing simultaneous redundant via insertion and line end extension. In the second stage, excess redundant vias are removed from those violating regions such that after the removal, the maximum via density rule is met while the total amount of redundant vias removed is minimized. This density-aware redundant via removal problem is formulated as a set of zero-one integer linear programming (0-1 ILP) problems each of which can be solved independently without sacrificing the optimality. The superiorities of our approaches are all demonstrated through promising experimental results.
\end{abstract}

\section{Introduction}

As the manufacturing technology shrinks, the feature size of a layout object becomes smaller but the scale of an integrated circuit (IC) becomes larger. However, the process variation becomes worse and damages the yield of an IC. In order to maintain manufacturability and high yield rates, a new design methodology, called design for manufacturability (DFM), is suggested [10][14]. To reduce the yield loss due to via failures is one of the most important issues in DFM.

A via in an IC layout provides the connection between two net segments from adjacent metal layers. The number of vias could become very large due to the design scale growing and/or the advent of the jumper-based solution to avoid the antenna effect [12]. Vias may fail partially or completely due to

* This work was partially supported by National Science Council under Grant No. NSC-95-2220-E-007-037, and Ministry of Economic Affairs under Grant No. MOEA-95-EC-17-A-01-S1-031.

Permission to make digital or hard copies of all or part of this work for personal or classroom use is granted without fee provided that copies are not made or distributed for profit or commercial advantage and that copies bear this notice and the full citation on the first page. To copy otherwise, to republish, to post on servers or to redistribute to lists, requires prior specific permission and/or a fee.

ICCAD'06, November 5-9, 2006, San Jose, CA

Copyright 2006 ACM 1-59593-389-1/06/0011...\$5.00 various reasons, such as cut misalignment and/or line-end shortening [14] during manufacturing processes. For a partially failed via, the contact resistance and the parasitic capacitance will increase and cause unexpected delay. On the other hand, a complete via failure will leave an open net in an IC layout and invalidate the functionality of the design.
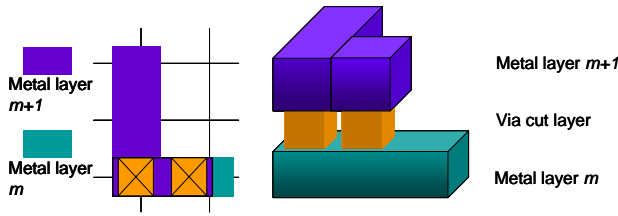

Fig. 1 Illustration for redundant via insertion.

One of the well known and highly recommended method to improve via yield/reliability is to add a redundant via adjacent to a single via [15][17]. Fig. 1 shows the top view and the 3D structure of a single via with a redundant via added to its right side. When a single via fails, its redundant via may still work; besides, the redundant via also provides an alternative signal path. Therefore, after adding a redundant via, the single-via failure can be tolerated and the whole via resistance can be also reduced.

The redundant via insertion problem can be considered in the routing or post-routing stage. The tools EYE/PEYE [5] consider redundant via insertion in the post-routing stage but the details of how they do redundant via insertion are not described. [13] and [4] also consider redundant via insertion in the post-routing stage. In [4], the single vias of a design are considered one by one to perform redundant via addition, and therefore the solution may just be locally optimal. Besides, since the approach will change the routing result of the timing noncritical nets, it may also induce timing violations even if designers keep the timing critical nets unchanged. [13] reduces the post-routing redundant via insertion problem into the maximum independent set (MIS) problem and proposes an effective heuristic to solve the MIS problem. The execution time of the approach, however, is generally longer according to the results reported in [13].

Both [6] and [7] consider redundant via insertion in the routing stage. [6] proposes a Lagrangian relaxation-based solution and [7] extends an existing multi-level routing framework to consider via minimization as well. However, postrouting ECO operations may change routing results and introduce extra vias into designs for the purposes of fixing timing, antenna or other problems. Therefore, no matter whether a router considers the redundant via insertion issue or not, it is usually necessary to perform redundant via insertion after the routing stage to further improve the yield and reliability of vias.

Among the set of via related design rules, the via density rules belong to the category of density control rules that arise for chemical-mechanical polishing (CMP) and other manufacturing steps which have varying effects on device and interconnect features depending on local layout density characteristics [1][16]. For each area of a pre-defined size on a via layer, its via density can be defined as the number of vias within it. If too many redundant vias are inserted into a die area, and exceed the maximum via density constraint, the pattern 
distortion of the vias in that area will become serious and hence the yield/reliability of the design will become worse. Therefore, after inserting redundant vias into a design, the maximum via density rule should be re-verified.

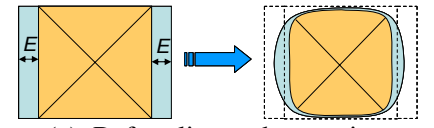

(a) Before line end extension

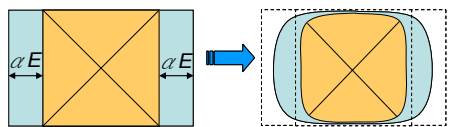

(b) After line end extension

Fig. 2. Via pattern distortions.

Another typical via related design rule is the via extension rule, which demands that, for a via, the portion of the connected metals must be extended beyond the edges of the via cut by at least $E \mu \mathrm{m}$ (see Fig. 2(a)), where $E$ is a process-dependent constant. With the extension distance, the slight cut misalignment during manufacturing can be tolerated. However, as the feature size of a layout object goes below the wavelength of the light used by optical lithography equipment, pattern distortions, such as line-end shortening, increase and aggravate the cut misalignment problem. As shown in Fig. 2(a), we can see that after line-end shortening, there is rare spacing to tolerate the cut misalignment phenomenon. Extending the lineends of metals for a via (i.e., line end extension) is another method for improving the via yield. Line end extension ${ }^{1}$ is to broaden the extension distance and thus can ease the cut misalignment problem caused by line-end shortening, as shown in Fig. 2(b).

In this paper, we study three via yield/reliability improvement problems in the post-routing stage. The first one is the redundant via insertion problem. For this problem, we accelerate the MIS-based approach proposed in [13] to solve it. The approach in [13] consists of the step of conflict graph construction followed by the step of solving an MIS problem on the graph. We present two methods to reduce the run time of the MIS-based approach by speeding up the conflict graph construction step and reducing graph size to facilitate the computation of an MIS solution, respectively. The experimental results indicate that the accelerated MIS-based approach is up to $3 \mathrm{X}$ faster without hurting solution quality. The second problem we consider in this paper is simultaneous redundant via insertion and line end extension. We formulate the problem as a maximum weighted independent set (MWIS) problem and enhance the accelerated MIS-based approach (which is originally designed only for the unweighted version) to solve it. Both steps of the accelerated MIS-based approach are modified to consider line-end extended vias as well. The experimental results indicate that the total number of inserted redundant vias and line-end extended vias is very close to the upper bound in each test case. The third problem is to simultaneously consider redundant via insertion and line end extension subject to the maximum via density rule, and a two-stage approach is presented to solve it. In the first stage, by ignoring the maximum via density rule, we enhance the MWIS-based approach to insert redundant vias and extend line ends as much as possible, and at the same time find the set of regions which violate the maximum via density rule. In the second stage, we remove redundant vias from those violating regions such that after the removal, the maximum via density rule is met while the total amount of redundant vias removed is minimized. This

\footnotetext{
${ }^{1}$ Because a fat via [4] will induce more capacitance and take more area than a line-end extended via, we choose to consider line-end extended vias in this paper.
}

density-aware redundant via removal problem is formulated as a set of zero-one integer linear programming (0-1 ILP) problems each of which can be solved independently without sacrificing the optimality. The experimental results indicate that our 0-1 ILP approach also runs efficiently.

To the best of our knowledge, the second and third problems have not been addressed before in the literature. Although an elegant approach based on the MIS formulation was recently proposed for the first problem [13], we are still able to incorporate novel speed-up methods into it.

The rest of this paper is organized as follows. In section II, we review the MIS-based approach given in [13] , and then describe two methods to improve its efficiency in section III. In section IV, we detail how to formulate the problem of simultaneous redundant via insertion and line end extension as a MWIS problem, and how to modify the accelerated MIS-based approach to solve the problem. In section V, the two-stage approach for solving the problem of simultaneous redundant via insertion and line end extension subject to the maximum via density rule is described. Section VI reports experimental results, and we conclude the paper in section VII.

\section{Redundant Via Insertion}

In this section, we review the problem formulation of postrouting redundant via insertion and the MIS-based approach given in [13]. Most of the notation and definitions are from [13].

\section{A. Redundant Via}

The manufacturing technology is assumed to consist of $2 m+1$ layers denoted by $M E_{1}, V I A_{1}, M E_{2}, V I A_{2}, \ldots, M E_{m}, V I A_{m}, M E_{m+1}$, where for all $i$ and $j, 1 \leq i \leq m+1$ and $1 \leq j \leq m, M E_{i}$, and $V I A_{j}$ represent the $i$ th metal layer and the $j$ th via layer, respectively. A via on $V I A_{i}$ involves the layers $M E_{i}, V I A_{i}$, and $M E_{i+1}$ and its position is specified by its center. We also assume that a set of via related design rules is given, and $S P$ is the spacing between two metals or via cuts ${ }^{2}$. We will use the symbols $X_{L L}(B)$ and $X_{U R}(B)\left(Y_{L L}(B)\right.$ and $Y_{U R}(B)$, respectively) to represent the $x$ coordinates ( $y$-coordinates, respectively) of the lower left and upper right corners of the bounding box ${ }^{3}$ of a layout object $B$, respectively.

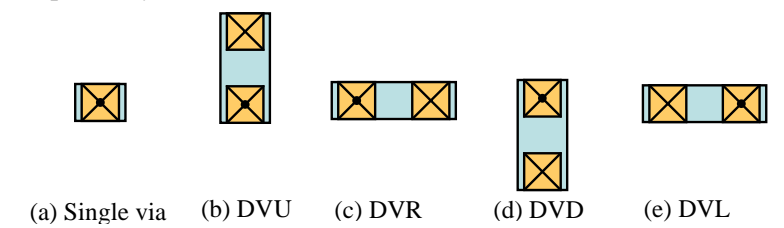

Fig. 3. Double via types.

A single via together with a redundant via inserted next to it is defined as a double via, and according to the position of a redundant via, a double via can be categorized into four types, as shown in Fig. $3^{4}$. (Note that in Fig. 3, each square with the $X$ symbol inside is called the via cut for a via.) Given a single via $i$, its double via of type $j(j \in\{D V U, D V D, D V L, D V R\})$ is denoted by $d v(i, j)$. Besides, a double via (or its corresponding redundant via) is said to be feasible if replacing the single via with it will not violate any design rule (excluding the maximum via density rule to be defined in section V), assuming none of

\footnotetext{
${ }^{2}$ Depending on the technology, the spacing between metals could be different from the spacing between via cuts. Also these spacing rules could vary on different layers. Nevertheless, all our approaches presented in this paper can be easily modified to handle all these cases.

3 The bounding box of an object in a design is the contour of its 2dimentional structure.

${ }^{4}$ The position of the single via is assumed to remain unchanged in each double via pattern. It should be mentioned that all our approaches presented in this paper can be easily extended to consider other double via patterns where the position of the single via is changed [4].
} 
the other single via has any redundant via inserted in the design. The problem of post-routing redundant via insertion is reduced to a maximum independent set problem (see Definition 1 and Problem 1) in [13].

Definition 1. (Conflict graph)

A conflict graph $G(V, E)$ is an undirected graph constructed from a detailed routing solution. For each single via $i$ on a signal net, if its double via of type $j(i . e ., d v(i, j))$ is feasible, there exists a vertex $v_{i, j}$ in $V$. An edge $\left(v_{i, j}, v_{i, j},{ }^{\prime}\right) \in E$ if and only if $i=i$, or $d v(i, j)$ and $d v\left(i, j^{\prime}\right)$ will cause design rule violations when both exist in the design.

Problem 1. Given a detailed routing solution, the problem asks to first construct a conflict graph from the design, then find a maximum independent set of the conflict graph, and finally for each vertex $v_{i, j}$ in the maximum independent set, replace the single via $i$ with the double via $d v(i, j)$.

To solve Problem 1, the MIS-based approach proposed in [13] consists of the step of conflict graph construction and the step of finding an MIS solution. In the next two subsections, we review the algorithm for conflict graph construction and the heuristic for finding an MIS solution, respectively.

\section{B. Conflict Graph Construction}

The conflict graph construction algorithm, called GCA, constructs the vertex set and edge set of a conflict graph simultaneously. The following definitions on DVE and DRW are from [13] and required for explaining GCA.

\section{Definition 2. (DVE)}

Suppose the bounding box of a single via $i$ is $R_{i}=\left[x_{i, l}\right.$, $\left.x_{i, u r}\right] \times\left[y_{i, l l}, y_{i, u r}\right]$ (see Fig. $4(a)$ ) and the bounding box of a double via $d v(i, j)$ is $R_{d v(i, j)}=\left[x_{d v, l l}, x_{d v, u r}\right] \times\left[y_{d v, l l}, y_{d v, u r}\right]$ (see Fig. 4 (b)). The reduced bounding box of $d v(i, j)$, denoted by $D V E(i, j)$, is defined as $R_{d v(i, j)}-R_{i}=\left[x_{e 1}, x_{e 2}\right] \times\left[y_{e 1}, y_{e 2}\right]$ (see Fig. 4 (c) for the illustration of $D V E(i, D V U))$.

\section{Definition 3. (DRW)}

Given a double via $d v(i, j)$, suppose the bounding box of the redundant via contained in $d v(i, j)$ is $R_{r v}=\left[x_{r 1}, x_{r 2}\right] \times\left[y_{r 1}, y_{r 2}\right]$. Then, the reduced design rule window of $d v(i, j)$ is defined to be $D R W(i, j)=\left[x_{r 1}-S P, x_{r 2}+S P\right] \times\left[y_{r 1}-S P, y_{r 2}+S P\right]$. (See Fig. $4(d)$ for the illustration of $D R W(i, D V U)$ which is the region with oblique lines.)

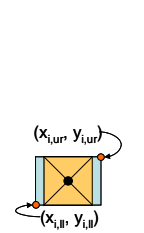

(a)

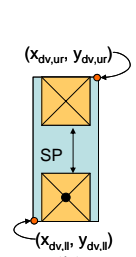

(b)

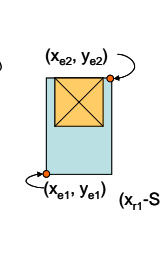

(c)
Fig. 4. Illustration of the DVE and the DRW for DVU

GCA first sorts all single vias by their $x$-coordinates in the non-decreasing order, and constructs an R-tree [2] for each metal layer to store the bounding boxes of all the layout objects on that layer. (Note that the bounding boxes of all single vias will be also stored in these R-trees) According to the sorted via sequence, denoted $1,2, \ldots, n$, each single via will be processed orderly as follows.

Suppose the single via being under consideration is $i$, where $1 \leq i \leq n$. For each double via $d v(i, j)$, where $j \in\{D V U, D V D, D V L$, $D V R\}, D R W(i, j)$ is used as the query window to do intersection range query on the R-trees of adjacent metal layers when checking if $d v(i, j)$ is feasible. If $d v(i, j)$ is feasible, the corresponding vertex $v_{i, j}$ will be added to the conflict graph.

To efficiently construct the edges in the conflict graph, GCA maintains a dynamically updated R-tree, called VNC (which is empty at the beginning), to store the DVE's for those feasible double vias which have been identified. Right before checking if each $d v(i, j)$ is feasible or not, for each element of $V N C$, if its right boundary is to the left of the left boundary of $D R W(i, D V L)$, it is impossible to overlap with any $D R W\left(i^{\prime}, j^{\prime}\right)$ for all $i^{\prime}, j^{\prime}$, with $i \leq i^{\prime} \leq n$ and $j^{\prime} \in\{D V U, D V D, D V L, D V R\}$, and therefore it will be deleted from $V N C$.

If $d v(i, j)$ is identified being feasible, $D R W(i, j)$ is again used as the query window to do intersection range query on $V N C$. For each $D V E\left(i\right.$ ', $\left.j^{\prime}\right)$ in $V N C$, if it intersects with $D R W(i, j)$, the edge $\left(v_{i, j}, v_{i}, j, j^{\prime}\right)$ will be added to the conflict graph. Finally, since a single via can only be replaced with one double via, GCA creates an edge for each pair of vertices each of which corresponds to a feasible double via of $i$. For each feasible double via $d v(i, j)$, its corresponding $D V E(i, j)$ is inserted to $V N C$.

\section{Heuristic for Finding an MIS Solution}

[13] presents a heuristic, called $H 2 K$, to find an MIS solution from a conflict graph. First, $H 2 K$ uses the feasible number and degree of a vertex as the first and second keys to construct a priority queue which stores all the vertices of a conflict graph. The feasible number of a vertex $v_{i, j}$ is defined to be the number of vertices $v_{i}, j$ 's in the conflict graph such that $i=i$ ' and $j \neq j$ ' (i.e., the number of the other feasible double vias originating from the same single via). A vertex has higher priority in the priority queue if it has smaller feasible number and degree.

Then $H 2 K$ finds an MIS solution in an iterative manner. At each iteration, a vertex subset of pre-defined size $k$ is extracted from the priority queue, and the subgraph induced by the vertex subset is obtained. Then, a maximal independent set solution on the subgraph is found (by any existing MIS solver) and added to the final solution. Finally, the conflict graph and priority queue are updated by removing those vertices appearing in the maximal independent set and their adjacent vertices and incident edges. Note that the feasible number of a vertex might become decreased in the updated conflict graph. $H 2 K$ will terminate when the conflict graph or priority queue has no remaining vertices.

\section{Methods for Speeding up the MIS-Based Approach}

In this section, we present two methods to speed up the MISbased approach. One is to accelerate the construction of a conflict graph and the other is to reduce the size of a conflict graph to which $H 2 K$ will be applied. They are detailed in the following two subsections.

A. Speed-up Method for Conflict Graph Construction

GCA constructs the R-tree of each metal layer statically right at the beginning to store the bounding boxes of all the original layout objects on the layer, and uses the R-trees of two adjacent metal layers for checking if a double via is feasible. Since a double via may induce design rule violations to a layout object only if they both locate in nearby grids, keeping in the R-trees the layout objects which are far enough from the single via being under consideration is not necessary. This implies that the run time of range queries on the R-trees has room to improve. In this subsection, we present a method to speed up GCA by dynamically maintaining an R-tree for each metal layer. We make the following modifications to GCA.

The R-tree of each metal layer is initially empty. For each metal layer, all its layout objects are sorted by the $x$-coordinates of the lower left corners of their bounding boxes in the nondecreasing order. Suppose via $i$ located at $\left(x_{i}, y_{i}\right)$ is the single via being under consideration. If none of the $x$-coordinates of the single vias that have been processed is equal to $x_{i}$, each R-tree will get updated as follows. Suppose $X_{L L}(d v(i, D V L))$ and $X_{U R}(d v(i, D V R))$ are equal to $x_{l l}$ and $x_{u r}$, respectively, as shown in Fig. 5. We first delete from each R-tree all the elements 
contained in the range $\left[-\infty, x_{l l}-S P\right] \times[-\infty,+\infty]$, such as $\mathrm{O}_{1}$ shown in Fig. 5, because they will not induce any design rule violation to all double vias of each single via which has not been processed by GCA yet. Then for each layout object $O B J_{R}$ remaining in the sorted order, if $X_{L L}\left(O B J_{R}\right)$ is less than $x_{l l}-S P$, it is removed from the sorted order; on the other hand, if $X_{U R}\left(O B J_{R}\right)$ is within the range $\left[x_{l l}-S P, x_{u r}+S P\right]$, it is removed from the sorted order and inserted into its corresponding R-tree. For each metal layer, this process is repeated until the first layout object $O B J_{F}$ with $X_{L L}\left(O B J_{F}\right)$ greater than $x_{u r}+S P$ (such as $\mathrm{O}_{2}$ shown in Fig. 5) is reached or no object remains in the sorted sequence.

With the above modifications, we can perform GCA to construct the vertex and edge sets of a conflict graph without keeping the whole layout objects of a design in the R-trees at all time. With possibly less layout objects stored in the R-trees, the range query time could be reduced. We name the modified conflict graph construction algorithm as IGCA.

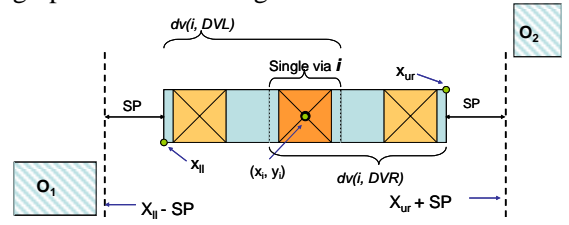

Fig. 5. The geometric information around a single via.

Fig. 6 illustrates how IGCA works. In Fig. 6(a), there is a layout consisting of seven wire segments, $\mathrm{O}_{1}, \mathrm{O}_{2} \ldots, \mathrm{O}_{7}$, and four vias, $\mathrm{V}_{1}, \mathrm{~V}_{2}, \ldots, \mathrm{V}_{4}$. Suppose the single via being under consideration is $\mathrm{V}_{3}$, and the layout objects $\mathrm{O}_{1}, \mathrm{O}_{2}$ and $\mathrm{V}_{1}$ will not induce any design rule violation to any double via of $\mathrm{V}_{3}$ or $\mathrm{V}_{4}$. For $G C A$, the R-trees for metal layers $m$ and $m+1$ will consist of all layout objects on the corresponding layers, as shown in Fig. 6(b). However, the layout objects $\mathrm{O}_{1}, \mathrm{O}_{2}$, and $\mathrm{V}_{1}$ are unnecessary when checking if a double via of $V_{3}$ is feasible; hence, the amount of layout objects stored in the R-trees for metal layers $m$ and $m+1$ is reduced by IGCA, as shown in Fig. 6(c).

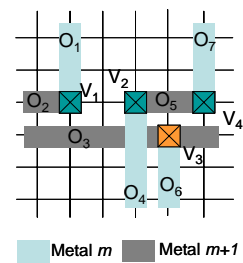

(a)

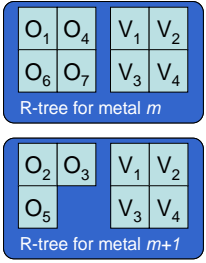

(b)

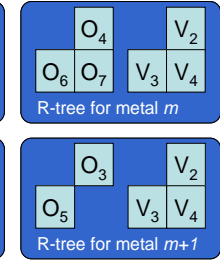

(c)
Fig. 6. Illustration for ICGA.

\section{B. Graph Reduction}

In this subsection, we present a graph reduction algorithm, called GRA, which selects and adds vertices into the final MIS solution during the construction of a conflict graph; as a result, the size of the conflict graph is reduced before $H 2 K$ is applied. Before describing the details of GRA, we need to define what internal and external edges are.

Definition 4. (Internal and external edges)

Given a conflict graph $G(V, E)$, an edge $e\left(v_{i, j}, v_{i, j},{ }^{\prime}\right) \in E$ is said to be internal if and only if $i=i$, and $j \neq j$, i.e., $v_{i, j}$ and $v_{i}, j$ ' correspond to two different double vias of the same single via. An edge is said to be external if it is not internal.

Suppose the single via $i$ located at $\left(x_{i}, y_{i}\right)$ is being under consideration by IGCA and none of the $x$-coordinates of the single vias that have been processed is equal to $x_{i}$. For each single via i' that has been processed by IGCA, if $X_{U R}\left(d v\left(i^{\prime}, D V R\right)\right)$ is less than or equal to $X_{L L}(d v(i, D V L))-S P$, and there is one vertex $v_{i}, j$ (corresponding to a feasible double via of the single via $i$ ') which is in the current conflict graph and has no external edge, then GRA will select $v_{i}$,j and add it to the final MIS solution. Since there will never be an external edge incident to $v_{i}, j$, inserting the double via $d v(i, j)$ will not cause any design rule violation or prevent any possible insertion of other doubles vias originating from single vias other than $i$ '. Finally GRA deletes all adjacent vertices of $v_{i, j}$ and all edges incident to those deleted vertices from the current conflict graph. As a result, $\mathrm{H} 2 \mathrm{~K}$ can solve the MIS problem on the reduced conflict graph without hurting the quality of the MIS solution.

Fig. 7 illustrates how GRA works. In Fig. 7(a), there are four single vias in the design, and they are numbered to form the sorted sequence. Suppose the single via 3 is being considered by $I G C A$ and its $x$-coordinate is different from the $x$-coordinates of single vias 1 and 2 . Besides, we also assume that $X_{U R}(d v(1, D V R))$ is less than or equal to $X_{L L}(d v(3, D V L))-S P$. The conflict graph right before adding the vertices corresponding to the feasible double vias of single via 3 is shown in Fig. 7(b), in which the bold edge connecting $\mathrm{V}_{1 \text {,DVR }}$ and $\mathrm{V}_{2 \text {,DVL }}$ stands for an external edge. Because there will be no new edge to be added for connecting any vertex corresponding to a feasible double via of single via 1 , either $\mathrm{V}_{1 \text {,DVu }}$ or $\mathrm{V}_{1, \mathrm{DVL}}$ (but not both) can be selected as an element of the final MIS solution immediately.

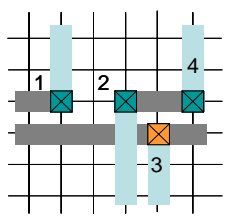

(a)

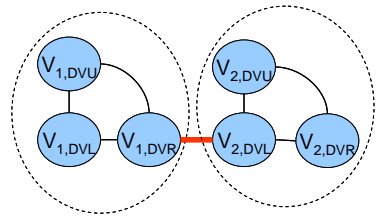

(b)
Fig. 7. Illustration for how GRA works.

\section{Overall Approach}

The accelerated MIS-based approach, which first uses IGCA and GRA to construct and reduce the conflict graph from a routed design, and then applies $H 2 K$ on the conflict graph, is named IMBA.

\section{Simultaneous Redundant Via Insertion and Line End Extension}

In this section, we first define the problem of simultaneous redundant via insertion and line end extension, and reduce it into a MWIS problem. We then describe how to modify the accelerated MIS-based approach, i.e., IMBA, to solve the problem.

\section{A. Line-end Extended Via}

We assume that the extension distance for a line-end extended via is $\alpha E$, where $E$ is the extension distance specified in the via extension rule for a single via, and $\alpha$ is a processdependent constant great than one. The structure of a line-end extended via is illustrated in Fig. 8. We use $L E$ to represent the line-end extended via structure.

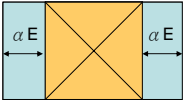

(a) Top view

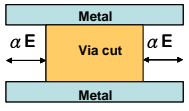

(b) Side view
Fig. 8. The structure of a line-end extended via.

\section{B. Problem Formulation}

The problem of simultaneous redundant via insertion and line end extension is defined as follows.

Problem 2. Given a detailed routing solution, without re-routing any signal net, the problem asks to replace single vias on signal nets with double vias or line-end extended vias such that after replacement, both the amounts of double vias and line-end extended vias are as large as possible. In addition, two conditions must be satisfied after replacement: First, each single via either remains unchanged, or is replaced by a double 
via or a line-end extended via. Second, no design rule is violated.

Extending the line end of a single via increases the manufacturability, but it also increases the via capacitance. On the other hand, although adding a redundant via next to a single via also increases the capacitance, it reduces the via resistance as well. Besides, redundant vias can improve not only yield but also reliability of a design. Therefore, adding a redundant via adjacent to a single via will has a higher priority than extending the line end of the single via in Problem 2.

In fact, we can view the line-end extended via structure $L E$ as a "pseudo" double via type. Given a single via $i$, let $d v(i, L E)$ denote its line-end extended via; $d v(i, L E)$ is said to be feasible if replacing $i$ with $d v(i, L E)$ will not violate any design rule, under the assumption that the other single vias remain unchanged. Given a single via $i$, both $D R W(i, L E)$ and $D V E(i, L E)$ are defined below.

Definition 5. (DVE and DRW for a line-end extended via)

Suppose the bounding box of a single via $i$ is $R_{i}=\left[x_{i, l l}\right.$, $\left.x_{i, u r}\right] \times\left[y_{i, l l}, y_{i, u r}\right]($ see Fig. 9(a)). The DVE(i, LE) and DRW $(i, L E)$ are defined as $\left[x_{i, l l}-(\alpha-1) E, x_{i, u r}+(\alpha-1) E\right] \times\left[y_{i, l l}, y_{i, u r}\right]$ (see Fig. $9(b))$ and $\left[x_{i, l l}-(\alpha-1) E-S P, \quad x_{i, u r}+(\alpha-1) E+S P\right] \times\left[y_{i, l l}-S P\right.$, $\left.y_{i, u r}+S P\right]$ (see Fig. $9(c)$ ), respectively.

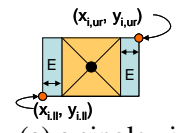

(a) a single via

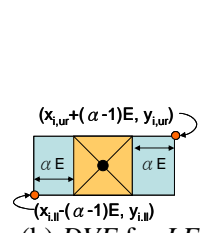

(b) DVE for $L E$

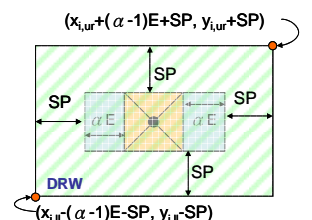

(c) $D R W$ for $L E$
Fig. 9. Illustration of $D V E$ and $D R W$ for $L E$.

The definition of a conflict graph is modified below to account for a weighted conflict graph which captures line-end extended vias as well.

Definition 6. (Weighted conflict graph)

A weighted conflict graph $G(V, E)$ is an undirected vertexweighted graph constructed from a detailed routing solution. For each single via $i$ on a signal net, if its double via of type $j$ $(j \in\{D V L, D V R, D V U, D V D, L E\})$ is feasible, there exists a vertex $v_{i, j}$ in $V$. An edge $\left(v_{i, j}, v_{i, j},\right) \in E$ if $i=i^{\prime}$, or $d v(i, j)$ and $d v\left(i^{\prime}, j^{\prime}\right)$ will cause design rule violations when both exist in the design. For each $v_{i, j}$ in $V$, if it corresponds to a line-end extended via, its weight is set to 1 ; otherwise its weight is set to $n+1$. Here $n$ is the number of vertices in $V$ each of which corresponds to a lineend extended via.

Given a weighted conflict graph, the MWIS problem is defined as follows. For each independent set of the graph, its cost is measured by the sum of the weights of all the vertices in the set. The MWIS problem asks to find an independent set with largest cost. It is not hard to prove that Problem 2 can be reduced to the MWIS problem. In the following subsection, we will describe how to enhance the accelerated MIS-based approach, i.e., IMBA, which combines ICGA, GRA, and $H 2 K$, to construct a weighted conflict graph and find an MWIS solution. We name this modified approach as EMBA.

\section{MWIS-based Approach}

Given a routed design, the corresponding weighted conflict graph $G(V, E)$ is constructed by ICGA and GRA with the following modifications. ICGA is modified such that it will treat each line-end extended via as a double via, and assign a weight to each vertex. This modified IGCA is named WIGCA. Since maximizing the amount of inserted redundant vias is the most important goal, GRA is modified in such a way that none of the vertices corresponding to feasible line-end extended vias is considered as a candidate for adding to the final independent set solution even if no external edge is incident to the vertex. This modified GRA is called WGRA. Let $M W I S_{1}$ be the set of vertices selected and added to the final solution by WGRA. The set $M W I S_{1}$ contains redundant vias only.

After $G$ is constructed, $H 2 K$ is applied to find a maximal weighted independent set $M W I S_{2}$ of $G$ with the following modifications. First, each vertex in the priority queue is added with the third key. If a vertex corresponds to a line-end extended via, it will have a lower priority on this key. With this modification, for vertices having the same feasible number and degree, the ones corresponding to redundant vias will be extracted first, and hence have higher chances to be included in the final solution. Second, a maximal weighted independent set is found from the extracted subgraph at each iteration. This modified $\mathrm{H} 2 \mathrm{~K}$ is called $\mathrm{WH} 3 \mathrm{~K}$.

Finally, the union of $M W I S_{1}$ and $M W I S_{2}$ is output as the final solution.

\section{Via-Density-Constrained Simultaneous}

\section{Redundant Via Insertion and Line End Extension}

In this section we first describe the via density rules and give the problem formulation of simultaneous redundant via insertion and line end extension under the maximum via density rule. We then detail our two-stage approach for solving the problem.

\section{A. Via Density Rules and Problem Formulation}

To analyze via density, each via layer $V I A_{i}$ is partitioned into a set $R(i)$ of overlapping rectangular regions each of which has the same width $W$ and height $H$, where $W$ and $H$ are processdependent constants. All the regions in $R(i)$ are organized into an $m$-row by $n$-column structure. See Fig. 10 for an illustration. We use $r(i, j, k)$ to represent the region which is in $R(i)$ and located at row $j$ and column $k$. For any two neighboring regions $r(i, j, k)$ and $r(i, j, k+1)$ in the same row, such as regions $A$ and $B$ in Fig. 10, their overlapped distance in the $x$-direction is defined to be $(1 / \lambda) W$, where $\lambda$ is a process-dependent constant. Similarly, for any two neighboring regions $r(i, j, k)$ and $r(i, j+1, k)$ in the same column, such as regions $A$ and $C$ in Fig. 10, their overlapped distance in the $y$-direction is defined to be $(1 / \beta) H$, where $\beta$ is a process-dependent constant. Therefore, a via is possible to be located in more than one region.

For each region $r(i, j, k)$, its via density, denoted by density $(i, j, k)$, is defined as the number of vias ${ }^{5}$ located in it. Two design rules related to via density, called the minimum via density rule and the maximum via density rule, can be considered. The minimum via density rule requires $\operatorname{density}(i, j, k)$ be greater than or equal to $L$ for each region $r(i, j, k)$, where $L$ is a process-dependent constant. On the other hand, the maximum via density rule requires $\operatorname{density}(i, j, k)$ be less than or equal to $U$ for each region $r(i, j, k)$, where $U$ is a process-dependent constant.

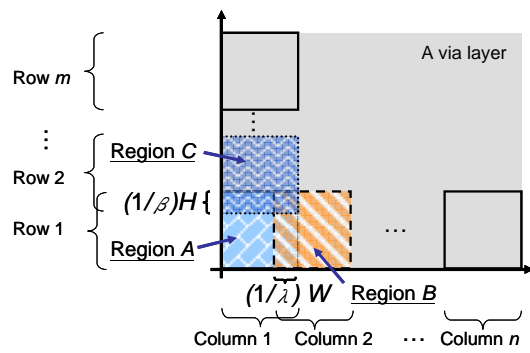

Fig. 10. Illustration for the via regions.

One method to meet the minimum via density rule is to add dummy vias [16] (if necessary), which unlike "normal” vias, are not used to provide signal paths between metal layers. In this paper, we assume the given routed design satisfies both the

\footnotetext{
${ }^{5}$ Each of such vias can be a single via, a redundant via, or a line-end extended via.
} 
minimum and maximum via density rules, and hence only the maximum via density constraint needs to be taken into account after adding redundant vias. Now Problem 2 is modified below to set Problem 3, while considers the maximum via density rule as well.

Problem 3. Given a detailed routing solution which already satisfies the minimum and maximum via density rules, without re-routing any signal net, the problem asks to replace single vias on signal nets with double vias or line-end extended vias such that after replacement, both the amounts of double vias and line-end extended vias are as large as possible. In addition, two conditions must be satisfied after replacement: First, each single via either remains unchanged, or is replaced by a double via or a line-end extended via. Second, no design rule, including the maximum via density rule, is violated.

\section{B. Overview of Our Two-stage Approach}

In this subsection, we give an overview of our two-stage approach for solving Problem 3. The details are described in the next two subsections.

In the first stage, the maximum via density rule is ignored, and we modify $E M B A^{6}$ such that in addition to performing simultaneous redundant via insertion and line end extension, it also reports the "violating" regions each of which violates the maximum via density constraint because of excess redundant vias inserted. Besides, for each violating region, the set of redundant vias that have been inserted in it, and the minimum number of redundant vias that must be deleted from it in order to meet the maximum via density rule are also generated.

In the second stage, we remove from each violating region redundant vias ${ }^{7}$ such that after the removal, the maximum via density rule is satisfied while the total amount of removed redundant vias is as small as possible. This redundant via removal problem will be formulated as a set of zero-one integer linear programming (0-1 ILP) problems each of which is solved independently without sacrificing the optimality.

In the next two subsections, we first describe how to modify $E M B A$ so as to efficiently find all the violating regions; we then explain the details of the 0-1 ILP approach.

\section{Via Density Calculation}

In this subsection, we describe how to extend EMBA to generate the information on each violating region, in addition to performing simultaneous redundant via insertion and line end extension.

For each region $r(i, j, k)$, let $\# s v(i, j, k)$ denote the number of single vias located in the region, and let $s v \_s e t(i, j, k)$ denote the set of single vias, each of which has at least one feasible redundant via (which may or may not be inserted into the design later) located in the region. It is not hard to see that a region $r(i, j, k)$ will not violate the maximum via density rule after adding redundant vias, if $\# s v(i, j, k)+\left|s v \_s e t(i, j, k)\right|$ is less than or equal to $U$. Let $D A N G E R=\left\{r(i, j, k)|\# s v(i, j, k)+| s v \_s e t(i, j, k) \mid>U\right.$, for all $i, j$ and $k$ \} be the set of regions which are possible to violate the maximum via density rule after redundant via insertion. For each feasible redundant via $v$ ', let reg_set( $\left.v^{\prime}\right)$ be the set of regions each of which is in DANGER and contains $v^{\prime}$. During the course of constructing the weighted conflict graph by WIGCA, we want to calculate \#sv(i,j,k), sv_set(i,j,k), DANGER and reg_set( $\left.v^{\prime}\right)$ as well. Therefore, we add the following extensions to WIGCA.

At the beginning, \#sv $(i, j, k)$ is 0 , and $s v \_s e t(i, j, k), \quad D A N G E R$ and reg_set $(v ')$ are all empty sets. For each via layer $V I A_{i}$, all its

\footnotetext{
${ }^{6}$ We can also discuss a variant of Problem 3, in which only redundant via insertion is considered. For this variant, we can modify IMBA and apply it in the first stage.

${ }^{7}$ Line-end extended vias will not be candidates for removal because each of them corresponds to a single via but with larger extension distance and therefore removing it will make a net become disconnected.
}

regions are sorted by the $x$-coordinates of their lower left corners in the non-decreasing order, and an R-tree is dynamically maintained to store its regions. These R-trees are initially empty. Suppose $v$ located at $\left(x_{v}, y_{v}\right)$ of the via layer $V I A_{i}$ is the single via being under consideration by WIGCA. If none of the $x$-coordinates of the single vias that have been processed is equal to $X_{v}$, the R-tree for each via layer will be updated as follows. Suppose $X_{L L}(d v(v, D V L))$ and $X_{U R}(d v(v, D V R))$ are equal to $X_{l l}$ and $x_{u r}$, respectively, as shown in Fig. 11. We first extract and delete from each R-tree all the regions contained in the range $\left[-\infty, x_{l l}\right] \times[-\infty,+\infty]$ (such as Region 1 shown in Fig. 11), because none of the single vias which have not been processed by WIGCA, and none of their feasible double vias are located in these regions. For each deleted region $r(i, j, k)$, if $\# s v(i, j, k)+\left|s v \_s e t(i, j, k)\right|>U$, we add $r(i, j, k)$ to both DANGER and reg_set $(v$ ') for each feasible redundant via $v$ ' that is contained in $r(i, j, k)$. Then for each region $r(i, j, k)$ remaining in the sorted order, if $X_{L L}(r(i, j, k))$ is less than $x_{u r}$, it is removed from the sorted order and inserted into the corresponding R-tree. For each via layer, this updating process is repeated on its R-tree until the first region $r\left(i^{\prime}, j^{\prime}, k^{\prime}\right)$ with $X_{L L}\left(r\left(i^{\prime}, j^{\prime}, k^{\prime}\right)\right)$ greater than $x_{u r}$ (such as Region 2 shown in Fig. 11) is reached or no object remains in the sorted order.

After updating the R-trees (if necessary), we use the via cut of $v$ as the query window to do range query on the R-tree corresponding to the via layer $V I A_{i}$ on which $v$ is located. For each queried region $r(i, j, k)$ that encloses the query window, we increase \#sv $(i, j, k)$ by one. Besides, for each feasible redundant via $v^{\prime}$ of $v$, we use its via cut as the query window to do range query on the corresponding R-tree as well; for each queried region $r(i, j, k)$ that encloses the query window, $v$ ' is found to be contained in $r(i, j, k)$, and if $v$ is not in $s v \_s e t(i, j, k)$ yet, we add $v$ to $s v \_s e t(i, j, k)$.

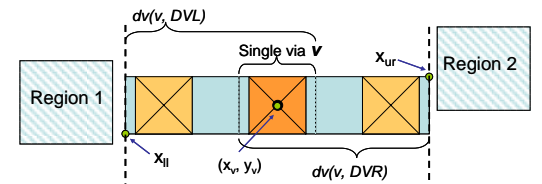

Fig. 11. Illustration for the dynamically updating strategy.

After all single vias of the design have been processed by WIGCA, for any remaining region $r(i, j, k)$ in each R-tree, if $\# s v(i, j, k)+\left|s v \_s e t(i, j, k)\right|>U$, we add $r(i, j, k)$ to both DANGER and reg_set( $v$ ') for each feasible redundant via $v^{\prime}$ that is contained in $r(i, j, k)$.

After applying $W H 3 K$ to perform simultaneous redundant via insertion and line end extension, let $R_{v i o}$ denote the set of all regions each of which violates the maximum via density rule; besides, for each region $r(i, j, k)$ in $R_{v i o}$, let $r v \_s e t(i, j, k)$ denote the set of all redundant vias that have been inserted into $r(i, j, k)$, and let $\# \operatorname{del}(i, j, k)$ be the minimum number of redundant vias that must be deleted from $r(i, j, k)$ in order to meet the maximum via density rule (i.e., density $(i, j, k)-\# \operatorname{del}(i, j, k)=U)$. $R_{\text {vio }}, r v \_s e t(i, j, k)$ and \#del $(i, j, k)$ are computed as follows. For each redundant via $v^{\prime}$ that has been inserted into the design, we add $v$ ' to $r v \_s e t(i, j, k)$ for each $r(i, j, k) \in$ reg_set $\left(v^{\prime}\right)$. For each region $r(i, j, k) \in D A N G E R$, if $\# s v(i, j, k)+\left|r v \_s e t(i, j, k)\right|>U$, it will be added to $R_{v i o}$, and $\#$ \#el $(i, j, k)$ will be set to \#sv $(i, j, k)+\left|r v \_s e t(i, j, k)\right|-U$.

\section{Redundant Via Removal}

When the first stage of our approach is done, $R_{\text {vio }}, r v \_s e t(i, j, k)$ and $\# \operatorname{del}(i, j, k)$ are ready for use in the second stage, for all $r(i, j, k) \in R_{\text {vio }}$. If $R_{\text {vio }}$ is empty, it means that the solution of simultaneous redundant via insertion and end line extension generated in the first stage already satisfies the maximum via density rule, and therefore nothing is done in the second stage. On the other hand, if $R_{v i o}$ is not empty, the following problem is solved in the second stage. 
Problem 4. Given $R_{\text {vio }}$, rv_set $(i, j, k)$ and \#del(i,j,k) for each $r(i, j, k) \in R_{v i o}$, the problem asks to remove redundant vias from each rv_set $(i, j, k)$ at least by the amount \#del(i,j,k) such that after the removal, each resulting region $r(i, j, k)$ satisfies the maximum via density rule while the total number of redundant vias removed is minimized.

We now describe a 0-1 ILP-based method to optimally solve Problem 4. Let $V E X=\bigcup_{r(i, j, k) \in R_{\text {vio }}} r v_{-} \operatorname{set}(i, j, k)$, be the set of redundant vias, say $r v_{1}, r v_{2}, \ldots, r v_{|V E X|}$, to be considered for removal. Problem 4 is formulated as a 0-1 ILP problem below.

$$
\begin{aligned}
& \text { Minimize } \sum_{r v_{p} \in V E X} X_{p} \\
& \text { s.t. } \\
& \sum_{r v_{p} \in r v_{-} \operatorname{set}(i, j, k)} X_{p} \geq \# \operatorname{del}(i, j, k), \forall r(i, j, k) \in R_{v i o} \\
& X_{p} \in\{0,1\}, \forall r v_{p} \in V E X
\end{aligned}
$$

where each redundant via $r v_{p}$ is associated with a zero-one variable $X_{p}$ and if $X_{p}$ is 1 , the redundant via $r v_{p}$ will be deleted from the design. The number of constraints and variables in the 0-1 ILP problem are equal to the number of regions violating the maximum via density rule, and the number of redundant vias located in these regions, respectively. For a large and/or high routing congestion design, the number of variables and/or constraints of the 0-1 ILP problem could become large.

In order to reduce the time complexity, we partition $R_{\text {vio }}$ into disjoint subsets $S u b_{1}, S u b_{2}, \ldots$, Sub $b_{t}$ (with $t \leq\left|R_{\text {vio }}\right|$ ) such that the following two conditions hold for each subset $S u b_{q}$ : (1) If there are two or more regions in $S u b_{q}$, then for each region $r(i, j, k) \in S u b_{q}$, there must exist a redundant via $r v$ and another region $r\left(i^{\prime}, j^{\prime}, k^{\prime}\right) \in S^{\prime} b_{q}$ such that $r v$ is contained in both regions $r(i, j, k)$ and $r\left(i^{\prime}, j^{\prime}, k^{\prime}\right)$. (2) For each region $r(i, j, k) \in S u b_{q}$, if a redundant via $r v$ is contained in $r(i, j, k)$, then $r v$ is not contained in any region in $R_{v i o}-S u b_{q}$. It is not hard to verify that we can individually formulate and solve a 0-1 ILP problem for each $\operatorname{Sub}_{q}$ without sacrificing the optimality. Besides, for each $S u b_{q}$, if it has only one region, we will arbitrarily delete the redundant vias from the region by the amount \#del $(i, j, k)$. The partitioning of $R_{\text {vio }}$ can be correctly done by the Union-Find algorithm [3].

\section{Experimental Results}

We used a $0.18 \mu \mathrm{m}$ technology which has 5 metal layers in our experiments. For simplicity we directly used the $\mathrm{R}^{*}$-tree package [11] for indexing 2-dimensional geometric information of layout objects. We used the qualex-ms [9] as the MIS or MWIS solver. Besides, we also limited the subgraph extracted at each iteration of $H 2 K$ or $M H 3 K$ to consist of 1500 vertices at most. Moreover, we used the lp_solver [8] as our 0-1 ILP solver. All the experiments were conducted on a Linux based machine with $2.4 \mathrm{G}$ processor and 4GB memory. All the run times are measured in seconds.

Table 1: The test cases

\begin{tabular}{|c|c|c|c|c|c|c|}
\hline Case & Size $(\boldsymbol{\mu m})$ & \#Nets & \#I/Os & \#Vias & \#Layers & \#Objects \\
\hline \hline C1 & $350.000 * 350.000$ & 4309 & 20 & 24594 & 5 & 218215 \\
\hline C2 & $419.433^{*} 413.28$ & 5252 & 211 & 41157 & 5 & 268669 \\
\hline C3 & $799.124 * 776.16$ & 18157 & 85 & 127059 & 5 & 933852 \\
\hline C4 & $691.272 * 680.400$ & 17692 & 415 & 151912 & 5 & 934073 \\
\hline C5 & $1383.482 * 1375.92$ & 44720 & 99 & 357386 & 5 & 2851612 \\
\hline
\end{tabular}

The test cases we used are the same as those in [13] and the detailed information is shown in Table 1; for each test case, the first column shows the circuit name, "Size $(\mu \mathrm{m})$ " gives the layout dimension, "\#Nets" shows the number of nets, "\#I/Os" gives the number of I/O pins, "\#Vias" shows the total number of single vias, and "\#Layers" gives the number of metal layers used. Finally, "\#Objects" gives the total number of layout objects including pins, vias, blockages and wire segments.
To see how much run time reduction could be achieved by our two speed-up methods, IGCA and GRA, we first focus on the problem of redundant via insertion. The results are shown in Table 2, where the columns "[13]", "IGCA+H2K" and "IMBA" are the redundant via insertion results after applying $H 2 K$ on the conflict graphs generated by GCA, IGCA, and IGCA+GRA, respectively. The column under "\#RV" gives the number of redundant vias inserted by the approach given in [13], and the column "\#diff" under each of "IGCA+H2K" and "IMBA" gives the difference between the number of redundant vias inserted by [13] and the number of redundant vias inserted by "IGCA+H2K" or "IMBA". Each column "T(s)" gives the run time of each approach, and the column "Speed-Up" under "IGCA+H2K" or "IMBA" gives the speed-up ratio of "IGCA+H2K" or "IMBA" over "[13]".

From Table 2, we can see that running IGCA alone is 1.98 2.46X (2.04X on average) faster than [13] while maintaining the same amount of redundant vias inserted for each test case. When we applied both IGCA and GRA, the speed-up ratio becomes even higher, ranging from 2.52 to 3 ; the average speed-up ratio is 2.92. Except for the test case C4, the total number of redundant vias inserted by IMBA for any other case is the same as that reported by [13]. The reason why IMBA inserted 2 less redundant vias for the test case C4 is that the size of the conflict graph was reduced before $H 2 K$ was applied, and $H 2 K$ could only guarantee to generate a maximal independent set (which is not necessarily a maximum independent set).

Table 2: Experimental results of [13], IGCA+H2K and IMBA

\begin{tabular}{||c||c|c||c|c|c||c|c|c|}
\hline \multicolumn{1}{|c||}{} & \multicolumn{2}{|c||}{$[13]$} & \multicolumn{3}{c||}{ IGCA+H2K } & \multicolumn{3}{c|}{ IMBA } \\
\cline { 2 - 9 } & \#RV & T(s) & \#diff & T(s) & $\begin{array}{c}\text { Speed- } \\
\text { Up }\end{array}$ & \#diff & T(s) & $\begin{array}{c}\text { Speed- } \\
\text { Up }\end{array}$ \\
\hline C1 & 17461 & 32 & 0 & 13 & $2.46 \mathrm{X}$ & 0 & 11 & $2.90 \mathrm{X}$ \\
\hline C2 & 28507 & 43 & 0 & 20 & $2.15 \mathrm{X}$ & 0 & 17 & $2.52 \mathrm{X}$ \\
\hline C3 & 91461 & 192 & 0 & 86 & $2.23 \mathrm{X}$ & 0 & 67 & $2.86 \mathrm{X}$ \\
\hline C4 & 101765 & 203 & 0 & 98 & $2.07 \mathrm{X}$ & -2 & 72 & $2.81 \mathrm{X}$ \\
\hline C5 & 254428 & 710 & 0 & 361 & $1.98 \mathrm{X}$ & 0 & 236 & $3.00 \mathrm{X}$ \\
\hline \hline Avg. & & $\mathbf{1}$ & & & $\mathbf{2 . 0 4 X}$ & & & $\mathbf{2 . 9 2 X}$ \\
\hline
\end{tabular}

For the problem of simultaneous redundant via insertion and line end extension, there is no existing approach. Therefore we develop a two-stage MIS-based approach called 2SMIS, and compare it with our approach EMBA. The main idea of 2SMIS is to insert redundant vias in the first stage, and then perform line end extension in the second stage. 2SMIS was implemented based on an extension of the MIS-based approach [13] by applying $H 2 K$ twice, one on a conflict graph consisting of vertices corresponding to redundant vias only, and the other on another conflict graph consisting of vertices corresponding to line-end extended vias only. Note that the number of redundant vias inserted by 2SMIS is always the same as that produced by the MIS-based approach [13].

In Table 3, the rows "2SMIS" and "EMBA" show the experimental results of the two approaches 2SMIS and EMBA, respectively. The column "Upp." denotes the number of single vias each of which has at least one feasible double via or one feasible line-end extended via. (Note that "Upp" can be thought of as an upper bound on the total number of redundant vias and line-end extended vias that can be inserted.) "\#RV" and "\#LE" show the numbers of redundant vias and line-end extended vias inserted, respectively. "R(\%)" gives the ratio of the sum of “\#RV” and "\#LE” (i.e., “\#RV” + “\#LE”) to "Upp.”. “T(s)” gives the CPU time of each approach. "Speed-Up" gives the speed-up ratio of "EMBA" over "2SMIS".

From Table 3, we can see that compared to 2SMIS, our approach $E M B A$ is able to insert the same (for three test cases) or almost the same (differs by 1 or 2 for the other two test cases) number of redundant vias, and more line-end extended vias (for all test cases). (Note that EMBA could insert less redundant vias because it is just a heuristic for the MWIS problem.) As a result, 
the total amount of redundant vias and line-end extended vias inserted by our approach is closer to the upper bound (the ratio ranges from $99.47 \%$ to $99.74 \%$ ) than $2 S M I S$. Besides, EMBA was 2.53 3.11X (2.92X on average) faster than 2SMIS.

Table 3: Experimental results of EMBA and 2SMIS

\begin{tabular}{|c|c|c|c|c|c|c|c|}
\hline Case & Upp. & & \#RV & \#LE & R(\%) & $T(s)$ & $\begin{array}{c}\text { Speed } \\
\text {-Up } \\
\end{array}$ \\
\hline \multirow{2}{*}{ C1 } & \multirow{2}{*}{18258} & 2SMIS & 17461 & 741 & 99.69 & 33 & \multirow{2}{*}{$2.53 \mathrm{X}$} \\
\hline & & EMBA & 17461 & 751 & 99.74 & 13 & \\
\hline \multirow{2}{*}{ C2 } & \multirow{2}{*}{29106} & 2SMIS & 28507 & 513 & 99.70 & 46 & \multirow{2}{*}{$2.42 \mathrm{X}$} \\
\hline & & EMBA & 28506 & 519 & 99.72 & 19 & \\
\hline \multirow{2}{*}{ C3 } & \multirow{2}{*}{93675} & 2SMIS & 91461 & 1946 & 99.71 & 206 & \multirow{2}{*}{$2.74 \mathrm{X}$} \\
\hline & & EMBA & 91461 & 1972 & 99.74 & 75 & \\
\hline \multirow{2}{*}{ C4 } & \multirow{2}{*}{104263} & 2SMIS & 101765 & 1917 & 99.44 & 217 & \multirow{2}{*}{$2.64 \mathrm{X}$} \\
\hline & & EMBA & 101765 & 1947 & 99.47 & 82 & \\
\hline \multirow{2}{*}{ C5 } & \multirow{2}{*}{261035} & 2SMIS & 254428 & 5730 & 99.66 & 814 & \multirow{2}{*}{$3.11 \mathrm{X}$} \\
\hline & & EMBA & 254426 & 5791 & 99.68 & 261 & \\
\hline & & & & & & & $2.92 \mathrm{X}$ \\
\hline
\end{tabular}

For the problem of simultaneous redundant via insertion and line end extension under the maximum via density rule, the width and height of each region on a via layer were set to $10.08 \mu \mathrm{m}$ and $8.4 \mu \mathrm{m}$, respectively, for each test case. Both $\lambda$ and $\beta$, defined in section $\mathrm{V}$-A, were set to 3 . For implementation simplicity, we used the center of a via to judge whether the via is located in a region. We observed that among all test cases, the maximum number of single vias located in a region was 30 , and therefore in order to enforce a very tight maximum via density rule in our experiments, the maximum number of vias, including single vias, redundant vias and line-end extended vias, that could be located in a region was set to 30 . The additional run times spent by the first stage of our approach on generating the information on violating regions were 2, 2, 18, 132, and 65 seconds for the five test cases, respectively. In the following discussion, we only focus on the redundant via removal problem.

Table 4. Statistics on the redundant via removal problem

\begin{tabular}{|c||c|c|c|c|}
\hline Case & $\begin{array}{c}\# \\
\text { Regions }\end{array}$ & $\begin{array}{c}\text { \#Vio. } \\
\text { Regions }\end{array}$ & $\begin{array}{c}\text { \#Sub- } \\
\text { prob. }\end{array}$ & $\begin{array}{c}\text { \#Cand. } \\
\text { rv }\end{array}$ \\
\hline \hline C1 & 52500 & 252 & 52 & 2238 \\
\hline C2 & 74000 & 412 & 90 & 3638 \\
\hline C3 & 257984 & 956 & 223 & 8478 \\
\hline C4 & 187200 & 9363 & 220 & 44396 \\
\hline C5 & 810816 & 1402 & 334 & 12648 \\
\hline
\end{tabular}

The input statistics are shown in Table 4. For each test case, the column "\#Regions" denotes the total number of regions. "\#Vio. regions" gives the number of regions violating the maximum via density rule, i.e., $\left|R_{\text {vio }}\right|$. "\#Sub-prob." shows the number of subsets which have more than one region, after the partitioning of $R_{\text {vio }}$ was done. (Note that "\#Sub-prob.” also specifies the number of 0-1 ILP problems to be solved.) "\#Cand. rv" is the total number of distinct redundant vias that are inserted in the regions of $R_{\text {vio }}$, i.e., $|V E X|$.

We also implemented two heuristics, called GREEDY and RANDOM, for comparative studies. GREEDY first creates a priority queue to store all the redundant vias in VEX. Each redundant via is associated with a key which is measured by the number of violating regions containing the redundant via. The method iteratively extracts from the priority queue a redundant via with the largest key, and deletes it from the design. Since deleting a vertex may cause the decrease of the keys for other redundant vias, the priority queue may need update after each deletion. As soon as no violating region exists, the method terminates. The second heuristic, RANDOM, iteratively selects a violating region randomly, and removes excess redundant vias from that region arbitrarily until there exists no violating region.

The number of redundant vias removed by each method and the run time are shown in the corresponding columns under "\# of removed rv" and "T(s)" of Table 5, respectively. The columns "0-1 ILP", "Greedy" and "Random" show the results of the 0-1 ILP methods (with and without doing partitioning), GREEDY and RANDOM, respectively. The columns "P" and "NP" under "T(s)" give the run times of both 0-1 ILP methods, respectively, where "P" stands for our method and "NP" is the one without doing partitioning on $R_{\text {vio }}$. It is clear to see that partitioning the whole $0-1$ ILP problem into a set of smaller problems helps to improve the run time for each test case; without doing partitioning, it is 6 times slower on average. Surprisingly our 0-1 ILP method with partitioning also runs faster than GREEDY for almost all test cases; on average GREEDY is 3.82 times slower. The reason why GREEDY is slower is mainly due to the overhead caused by updating the priority queue after each via removal. For each test case, although RANDOM runs much faster than our method, the amount of redundant vias removed by it is also much larger. Similarly GREEDY also removed more redundant vias than our method. On average, GREEDY and RANDOM are 1.5\% and $29.6 \%$ worse in terms of the number of redundant vias removed.

Table 5: The performance comparison

\begin{tabular}{|c||c|c|c||c|c|c|c|}
\hline \multirow{2}{*}{ Case } & \multicolumn{3}{|c||}{ \# of removed rv } & \multicolumn{4}{c|}{ T(s) } \\
\cline { 2 - 8 } & \multirow{2}{*}{$\mathbf{0 - 1}$ ILP } & \multirow{2}{*}{ Greedy } & \multirow{2}{*}{ Random } & $\mathbf{0 - 1}$ ILP & \multirow{2}{*}{ Greedy } & \multirow{2}{*}{ Random } \\
\cline { 5 - 8 } & & & & P & NP & & \\
\hline C1 & 387 & 389 & 486 & 0.03 & 0.09 & 0.03 & $<0.01$ \\
\hline C2 & 625 & 627 & 748 & 0.05 & 0.22 & 0.08 & $<0.01$ \\
\hline C3 & 1501 & 1502 & 1762 & 0.13 & 1.32 & 0.89 & $<0.01$ \\
\hline C4 & 10631 & 10856 & 14379 & 14.3 & 84.0 & 52.2 & 0.04 \\
\hline C5 & 2370 & 2373 & 2732 & 0.26 & 3.11 & 3.25 & 0.01 \\
\hline \hline Avg. & $\mathbf{1}$ & $\mathbf{1 . 0 1 5}$ & $\mathbf{1 . 2 9 6}$ & $\mathbf{1}$ & $\mathbf{6 . 0 0}$ & $\mathbf{3 . 8 2}$ & $\mathbf{0 . 0 0 3}$ \\
\hline
\end{tabular}

\section{Conclusions and Future Works}

In this paper, we have studied three post-routing via yield/reliability improvement problems, and have presented novel approaches for them. The experimental results well support all our approaches. Our future work is to study how to consider redundant via insertion, line end extension, and via density rules all together in one single stage.

\section{References}

[1] A. B. Kahng, "Research Directions for Coevolution of Rules and Routers", Proc. of ISPD, 2003.

[2] A. Guttman, "R-Trees: A Dynamic Index Structure for Spatial Searching", Proc of SIGMOD, 1984.

[3] Cormen, Leiserson and Rivest, Introduction to Algorithms (Second Edition), The MIT Press, 2001.

[4] F. Luo, Y. Jia and W.-M. Dai, "Yield-Preferred Via Insertion Based on Novel Geotopological Technology”, Proc. of ASP-DAC, 2006.

[5] G. A. Allan, "Targeted Layout Modifications for Semiconductor Yield/Reliability Enhancement", IEEE Trans on Semiconductor Manufacturing, vol. 17, Nov. 2004.

[6] G. Xu, Li-Da Huang, D. Z. Pan and M. D. F. Wong, "Redundant-Via Enhanced Maze Routing for Yield Improvement”, Proc. of ASP-DAC, 2005.

[7] H. Yao, Y. Cai, X. Hong and Q. Zhou, "Improved Multilevel Routing with Redundant Via Placement for Yield and Reliability", Proc. of GLSVLSI, 2005.

[8] http://lpsolve.sourceforge.net/

[9] http://www.busygin.dp.ua/npc.html

[10] L. K. Scheffer, "Physical CAD Changes to Incorporate Design for Lithography and Manufacturability”, Proc. of ASP-DAC, 2004.

[11] N. Beckmann, H.-P. Kriegel, R. Schneider and B. Seeger, "The R*-Tree: An Efficient and Robust Access Method for Points and Rectangles", Proc. of SIGMOD, 1990.

[12] P. H. Chen, S. Malkani, C.-M. Peng and J. Lin, "Fixing Antenna Problem by Dynamic Diode Dropping and Jumper Insertion“, Proc. of ISQED, 2000.

[13] K.-Y. Lee and T.-C. Wang, "Post-Routing Redundant Via Insertion for Yield/Reliability Improvement”, Proc. of ASP-DAC 2006.

[14] S. Raghvendra and P. Hurat, "DFM: Linking Design and Manufacturing", Proc. of ICVD, 2005.

[15] TSMC Reference Flow 5.0.

[16] V. Pitchumani, B. Landau and J. Brandenburg, Design for Manufacturability : Embedded Tutorial, ASP-DAC 2005.

[17] Y. Zorian, D. Gizopoulos, C. Vandenberg and P. Magarshack, "Guest Editors' Introduction: Design for Yield and Reliability”, IEEE Trans on Design \& Test of Computers, vol. 21, May 2004. 